\title{
(Neo-)Marxistische Politische Ökonomie als Gegenprogramm zur Standardökonomik: Kommentar zum Beitrag von Hardy Hanappi
}

\author{
Frank Beckenbach
}

Online publiziert: 27. Februar 2019

(C) List-Gesellschaft e.V. 2019

\section{Einleitung}

Hanappi (2019) versucht in seinem Beitrag die Besonderheit des Denkens von Marx durch seine biografische Entwicklung in Gestalt seiner politischen ebenso wie philosophischen Prägung zu charakterisieren.

Die dort entstandenen eher intuitiven Orientierungen sieht er als Grundlage für die auf Basis der gescheiterten Revolutionen erfolgende Hinwendung von Marx zum Studium der zeitgenössischen Politischen Ökonomie. In diesem Studium sieht er folgende Problemfelder als virulent an:

- das Problem der Aggregierung unterschiedlich produktiver und qualifizierter individueller Arbeiten,

- den Antagonismus zwischen dem Entwicklungsstand der Produktivkräfte und den Produktionsverhältnissen,

- die Entfremdung der ökonomischen Akteure gegenüber den gesellschaftlichen Bedingungen, in denen sie operieren und schließlich

- die Frage, in welchen Bewusstseinsformen sich diese gesellschaftlichen Verhältnisse sich bei den Akteuren ausdrücken.

Die Fokussierung auf diese Problemfelder führt den Autor zu einer spezifischen Auswahl und Präsentation der durch Marx inspirierten Denkrichtungen: den ,Matrix-Marxismus“, den „Makroökonomischen Marxismus“, den ,,evolutionstheoretischen Marxismus“ und schließlich den „synthetischen Marxismus“. In Bezug auf

This commentary article refers to the article available online at https://doi.org/10.1007/s41025-01900127-6. An author's reply to this commentary article is available online at https://doi.org/10.1007/ s41025-019-00147-2.

F. Beckenbach $(\bowtie)$

Cusanus Hochschule, Bernkastel-Kues, Deutschland

E-Mail: beckenbach@wirtschaft.uni-kassel.de 
diese Denkrichtungen wird dann jeweils genauer diskutiert, ob und wenn ja, welche Elemente für eine Kritik der Standardökonomik beigebracht werden können. Dies führt den Autor auch zu interessanten aber weitläufigen Überlegungen zu wissenschaftspolitischen, zeitgeschichtlichen und politischen Entwicklungen die eingestandenermaßen über die gesetzte Themenstellung hinausgehen. ${ }^{1}$ Diese Erörterungen der Wirkung von Marx und des Marxismus schließen mit der Bemerkung: „Wenn das präsentierte Gedankengut in so vielen Bereichen Eingang gefunden hat und zu einer Selbstverständlichkeit geworden ist mit der die praktizierenden Forscher umgehen ohne auch nur mehr zu ahnen von wem es stammt, dann hat der Gelehrte seine individuelle Rolle in vollem Umfang erfüllt und diese ist im allgemeinen Wissen aufgegangen - oder mit Hegel: aufgehoben." (Hanappi 2019, S. 22) Angesichts der damit verbundenen zahlreichen ,stille Post“-Prozesse möchte ich in diesem Kommentar das Bewusstsein für die Besonderheit des theoriegeschichtlichen Ausgangspunktes in Gestalt des hinterlassenen ökonomietheoretischen Werks von Marx schärfen und dessen Brauchbarkeit für eine Kritik der heutigen Standardökonomik genauer diskutieren.

Vor diesem Hintergrund erscheint mir weder die Auswahl der genannten Problemfelder geeignet, noch die Auswahl der genauer diskutierten „Marxismen“. Aufgrund der damit verbundenen partialanalytischen Fragmentierung des ökonomietheoretischen Gesamtkonzepts von Marx einerseits und der mehr oder weniger starken (auch von Hanappi (2019) eingestandenen) Prägung dieser „Marxismen“ durch die Standardökonomik andererseits erscheint es daher geboten, sich zunächst (nochmal) der wichtigsten ökonomietheoretischen Aussagen von Marx in seinem „Spätwerk“ zu vergewissern ${ }^{2}$ und auf dieser Grundlage die Kritikimplikationen des Marxschen Ansatzes für die heutige Standardökonomik deutlich zu machen (Abschnitt 2). Die modelltheoretisch vorgetragenen Versuche zur Überbrückung dieser Differenz können dann als fragwürdige ,moderne“ Rekonstruktionen problematisiert und angemessene Forschungsbedarfe spezifiziert werden (Abschnitt 3).

\section{2 (De-)Konstruktive Rückbesinnung auf die Marxsche Kritik der Politischen Ökonomie}

Die (de)konstruktive Rückbesinnung auf das Marxsche Konzept der „Kritik der Politischen Ökonomie“" soll im Folgenden zunächst (A) durch Vergewisserung über die besonderen Merkmale seines Ansatzes erfolgen und dann $\left(\mathrm{A}^{\prime}\right)$ dessen kritische Differenz zum Ansatz der Standardökonomik deutlich machen. Im Anschluss daran

\footnotetext{
${ }^{1}$ Dies gilt v.a. für die Vebindung von marxistischen und keynesianischen Theorieelementen zu neuen Theoriekonstrukten (vergl. Hanappi 2019, S. 5 ff.) aber auch für die Nutzbarmachung des Leontiefschen Input-Output Schemas zur Erklärung des Übergangs vom Feudalismus zum Kapitalismus (vergl. Hanappi 2019, S. 8 ff.).

2 Ich interpretiere dieses Werk nicht so, dass ,(n)ach der Veröffentlichung des ersten Bandes von „Das Kapital“ “.. Marx intensiv damit beschäftigt (ist - F.B.) die dort eher intuitiv und konzentriert versammelten Ideen vertieft und mit breiterem historischen Hintergrund zu fundieren." (Hanappi 2019, S. 5). Vielmehr lassen alle bisher veröffentlichten Manuskripte trotz aller Fragmentierungen ein systematisches Grundkonzept erkennen.
} 
wird (B) die Besonderheit seiner werttheoretischen Orientierung und $\left(\mathrm{B}^{\prime}\right)$ deren Relevanz für eine Kritik der Standardökonomik thematisiert. Auf dieser Grundlage wird (C) die Bedeutung der Marxschen Klassentheorie der Produktion und die damit zu verbindende endogene Erklärung des technischen Wandels aufgegriffen und wiederum $\left(\mathrm{C}^{\prime}\right)$ mit der Standardökonomik kontrastiert. Die diese Elemente aufnehmende ökonomische Entwicklungstheorie (D) wird kritisch gegen die Standardökonomik gewendet $\left(\mathrm{D}^{\prime}\right)$. Schließlich sollen die Umrisse des Marxschen Wettbewerbskonzepts (E) im Blick auf die Standardökonomik diskutiert werden $\left(\mathrm{E}^{\prime}\right)$.

\subsection{Begriffliche Entwicklung als wissenschaftlicher Erklärungstyp}

(A) Generell liegt dem Marxschen Ansatz eine duale Betrachtungsweise zugrunde: sie beruht auf einer Unterscheidung und der Kombination universaler (überhistorischer) Eigenschaften (Ebene des Gebrauchswerts in seinen Ausdifferenzierungen) einerseits und einer historisch besonderen Ausprägung derselben (Ebene des Tauschwerts und dessen Fortentwicklung) andererseits. Der Kern der von Marx verfolgten wissenschaftlichen Erklärung besteht dabei in einer schrittweisen Konkretisierung von zunächst abstrakten begrifflichen Unterscheidungen (begriffliche Entwicklung). Diese Konkretisierung soll bis zu beobachtbaren Sachverhalten vorangetrieben werden. Damit ist ein Erklärungstyp anvisiert der - modern gesprochen - jenseits des Positivismus und diesseits des Konstruktivismus liegt. Durch den sich erst am Ende dieser Darlegung einstellenden Bezug auf beobachtbare „Erscheinungen“" soll ein empirischer Geltungsanspruch für das System als Ganzes (und damit auch für seine „abstrakten“ - nicht beobachtbaren - Wesensmerkmale) hergestellt werden. Insofern verfolgt Marx ein wissenschaftliches Entschlüsselungs- und Aufklärungsprogramm über nicht-erfahrbare Sachverhalte incl. der Rechenschaftslegung darüber, wie dies überhaupt möglich ist.

Inhaltlich ist das übergreifende Thema dieses Konzepts der „Kapitalkreislauf“: das Prozessieren des Einzel- wie des Gesamtkapitals durch seine verschiedenen Existenzformen. Entsprechend des im vorangegangenen Abschnitt charakterisierten Vorgehens wird dieser Kapitalkreislauf mit unterschiedlichen Fokussierungen und auf unterschiedlichen Abstraktionsebenen analysiert. Marx legt insoweit eine dynamische Systemanalyse vor mit dem Ziel, die Elemente dieses Systems transparent zu machen und ihre konfliktorischen Beziehungen zu verdeutlichen. Akteure tauchen in diesem Systemkontext nur als „Personifikationen“ auf: sie sind Vollstrecker von Systemerfordernissen. Insofern kann aus moderner Sicht dieses Vorgehen mit der Frage konfrontiert werden, wie diese systemischen (Makro-)Zusammenhänge durch Akteurshandeln fundiert werden können (Makro $\rightarrow$ Mikroproblem). ${ }^{3}$

Methodisch gesehen besteht das Konzept von Marx in den von ihm selbst noch autorisierten Veröffentlichungen bzw. in den nunmehr posthum weitgehend veröffentlichten Manuskripten aus Theoriefragmenten. Diese sind für sich jeweils unfertig und untereinander nicht vollständig abgestimmt. Insofern lassen sich nur Umrisse

\footnotetext{
3 Diese Frage drängt sich nicht nur wegen der Orientierung des überwiegenden Teils der Standardökonomik auf, sondern auch wegen der mit der fehlenden akteurstheoretischen Fundierung zusammenhängenden Erklärungslücken im Marxschen Konzept selber (vergl. Goldstein 2006).
} 
eines Hypothesengerüsts und damit zusammenhängender Kausalitäten identifizieren. Nicht nur diese Unvollendetheit der Theorie, sondern auch die Unentwickeltheit entsprechender Instrumente sind die Ursache dafür, dass nur Rudimente einer Modellierung (in Gestalt der Verwendung abstrakter Sprachelemente bzw. mathematischer Symbole) von Marx hinterlassen wurden.

$\left(\mathrm{A}^{\prime}\right)$ Demgegenüber kann die ,Standardökonomik“ als ein vielschichtiges aber zusammenhängendes Forschungs- und Lehrprogramm charakterisiert werden, das sich erst nach dem 2. Weltkrieg herausgebildet hat (vergl. Beckenbach 2017). Dieses Forschungs- und Lehrprogramm besteht einerseits aus dem gemeinsamen Kern, die Allokation knapper Ressourcen als Zentralfragestellung ökonomisch zu spezifizieren entlang einer als Maximierung unter Beschränkungen gefassten ,rationalen“ Handlungslogik, deren gesellschaftliche Kompatibilität entlang von Gleichgewichtskonstruktionen modelltheoretisch postuliert wird. Andererseits gibt es sowohl für diese Rationalitätsnorm als auch für die Gleichgewichtsspezifikation Varianten innerhalb dieses gemeinsamen Kerns (vergl. Beckenbach et al. 2016, S. 58 ff.). Nicht zuletzt aufgrund der in den ,modernen“ Wissenschaften Platz greifenden Performanzorientierung besteht aber eine Tendenz zu einer dichteren Anbindung der verschiedenen Themengebiete an den o.g. gemeinsamen Kern und damit insbesondere auch zu einer auf dieser Grundlage erfolgenden Verbindung von Mikro- und Makroökonomik.

In diesem Forschungs- und Lehrprogramm gibt es entsprechend der genannten Zentralfragestellung keine Unterscheidung zwischen universalen (überhistorischen) und historisch-spezifischen Sachverhalten. Elemente der letzteren werden (eher implizit als explizit) in die Betrachtung der ersteren projeziert (z. B. die Knappheit der Ressourcen und der Individualismus/homo oeconomicus werden als überhistorische Phänomene angesehen). Aufgrund der Anbindung der Erkenntnisgewinnung an die mit dem gemeinsamen Kern umschriebene Sichtweise gibt es eine markante Theoriearchitektur sowohl bei der Beantwortung der grundsätzlichen Forschungsfragen als auch in den Spezialisierungsgebieten. Diese Theoriearchitektur wird flankiert durch eine in mathematischer Form erfolgende Modellbildung (spezielle Form des mathematisch konstruierten Wissens). Insofern liegt mit der Standardökonomik aus der Sicht des Marxschen Ansatzes ein modelltheoretisch fundiertes überhistorisches Legitimationsprogramm für historisch-empirisch konnotierte Sachverhalte vor.

Inhaltlich ist das übergreifende Thema dieses Konzepts der „Konsum“ (als Endzweck der Produktion) und die auf die entsprechenden Handlungsoptionen bezogenen „Präferenzen“ (als individuelle Richtschnur für das Handeln). Dabei werden diese Präferenzen so modelliert, dass - je nach der Spezifikation des Handlungskontexts - deren größtmöglich Kompatibilität in Gestalt eines Gleichgewichts konstruiert werden kann. Entgegen der Marxschen Sichtweise fokussiert die Standardökonomik damit einen Zustand der gesellschaftlichen Kompatibilität individueller Handlungen und daher einen Zustand gesellschaftlicher Harmonie (bzw. einen gesellschaftlichen Glückszustand). Ganz anders als bei Marx liegt hier entsprechend eine transparente Instrumentalisierung ökonomischer Aktivitäten und Institutionen für individuelle Ziele und gesellschaftliche Wohlfahrtszwecke vor. Akteure sind in diesem Theorie- und Modellkontext algorithmisierte Persönlichkeiten: sie sind Vollstrecker von Zielerfordernissen entlang vorgegebener Kalkulationsregeln. Dieses Vorgehen kann entsprechend mit der Frage konfrontiert werden, wie diese auf 
sich selbst gestellten Akteure ohne einen zentralen Planer die in den Gleichgewichtskonstruktionen formulierten Koordinationsleistungen auch erbringen können. Invers zu Marx gibt es daher in diesem Kontext trotz aller Integration der Teilgebiete ein Mikro $\rightarrow$ Makroproblem.

\subsection{Entschlüsselung der Verdinglichung gesellschaftlicher Verhältnisse}

(B) Der „Wert“ ist eine Grundkategorie in dem unter (A) beschriebenen wissenschaftlichen Entschlüsselungsprogramm. Entsprechend ist er nicht unmittelbar erfahrbar, sondern setzt sich ,hinterrücks“ in den gesellschaftlichen Aktivitäten durch. In der Marxschen Sicht umschreibt der Wert die Grundform für die Herstellung des gesellschaftlichen Zusammenhangs im Kapitalismus. Diese ist dadurch charakterisiert, dass die Akteure sich einerseits in gesellschaftlicher Abhängigkeit befinden (manifest etwa in Gestalt der Teilung der Arbeit) und andererseits ihre Aktivitäten privaten Charakter haben (was in der Folge(!) dann in Gestalt von Eigentumsrechten abgesichert wird). Die „Wertform“ des Produkts dieser Privataktivitäten ist dann gleichbedeutend mit der Artikulation eines gesellschaftlichen Anerkennungsbedarfs. Dabei wird dieser Anerkennungsbedarf als Eigenschaft des Produktes ausgedrückt. Unter den Bedingungen privater arbeitsteiliger Produktion kann dieser Anerkennungsbedarf nur durch Bezug auf ein allgemein anerkanntes Medium, dem Geld erfolgen. Die allgemeine Wertform ist daher die Preisform. Diese mit der Kategorie des Werts verbundene Erfassung der historisch-spezifischen Form, in der sich in der kapitalistischen Produktionsweise die gesellschaftliche Synthese der diversen privaten Aktivitäten herstellt, gerät aber schnell aus dem Blick wenn damit nur ein „Aggregationsproblem“ (Hanappi 2019, S. 3) verbunden wird.

Gesellschaftliche Praktiken verwandeln sich auf diese Weise in Sachgesetzlichkeiten und unterwerfen die Akteure. Ergo: der gesellschaftliche Zusammenhang stellt sich indirekt in „,verdinglichter“ Form her. ${ }^{4}$ Marx verwendet in diesem Zusammenhang die Fetischismusmetapher: die potenzielle gesellschaftliche Anerkennung scheint für die Gesellschaftsmitglieder der Ware und noch mehr dem Medium des Geldes als dingliche Eigenschaft innezuwohnen obwohl sie doch eigentlich erst in einem spezifisch historischen Kontext solche Eigenschaften annehmen. ${ }^{5}$ Damit geht einher, dass diese verdinglichte Form der gesellschaftlichen Anerkennung erst nach der Produktionsaktivität erfolgt und insoweit prekär ist. Ein Erfolg ist nicht garantiert. Vor diesem Hintergrund ist die Marxsche Kapitalismusanalyse eine Entwicklung der Formen des Werts: Ware, Geld, Kapital, Lohn, Profit und Rente sind dann

\footnotetext{
${ }^{4}$ Dieser Teil der späten Marxschen Ökonomietheorie kann als eine Fortführung und Präzisierung des Entfremdungskonzepts aus den Frühschriften angesehen werden. Hanappi (2019) hält sich überwiegend an die letzteren und sieht daher die Notwendigkeit einer sozialpsychologischen Erweiterung der Theorie (Hanappi 2019, S. 3 f., 7).

${ }^{5}$ Hanappi (2019) weist zu Recht darauf hin, dass diese Diagnose eines verkehrten Bewußtseins auch für die strukturell benachteiligten gesellschaftlichen Gruppen gilt und insoweit ein soziales Veränderungspotential dadurch blockiert werden kann (Hanappi 2019, S. 4, 22).
} 
gedankliche Stationen der prekären Verselbständigung und Verdinglichung dieser gesellschaftlichen Anerkennungsprozeduren (vergl. Marx 1992, S. 848 ff.). ${ }^{6}$

Diese auf die spezifisch historische Form des Kapitalismus zielende werttheoretische Orientierung ist nicht an die speziellen Hypothesen gebunden, die Marx in diesem Kontext entwickelt:

- Marx leitet aus der Analyse des „Warentauschs“ die Hypothese ab, dass die in den getauschten Waren enthaltenen „,notwendigen“ Arbeitsmengen die Preise und damit den Tausch regulieren. Weder die entsprechenden Ausführungen im „Kapital“ (vergl. Marx 1987, S. 70ff.; zur Kritik: Beckenbach 2018, S. 58f.) noch die von Marx - nicht zuletzt im Blicke auf höhere Gesellschaftsorganisationen - vorgenommene Postulierung eines „Naturgesetzes“ der Regulierung durch Arbeitszeiten (vergl. Marx 1974, S. 552 f und Marx 1969, S. 18 ff.) machen aber diese Hypothese plausibel (vergl. die frühe etwas dogmatische Kritik bei Lippi 1979). ${ }^{7}$

- Der werttheoretischer Ansatz ist auch nicht an die Hypothese von Marx gebunden, dass das Spezifikum des Kapitalismus darin besteht, dass hier die Warenproduktion verallgemeinert sei sodass derselbe als geschlossenes System der Warenreproduktion mittels Waren behandelt werden kann. ${ }^{8}$ Vielmehr zeigt die Durchführung der Marxschen Kapitalismusanalyse, dass die mit dem Kapitalkreislauf verbundenen Konflikte (etwa bei der Geldorganisation, bei der Länge des Arbeitstags und der Gestaltung der Arbeitsbedingungen) staatliche Absicherungen bei der mit der „Zirkulation“ (als der Sphäre der Herstellung des gesellschaftlichen Zusammenhangs) verbundenen Legitimationen erfordern (vergl. Beckenbach 2018, S. 58 ff.). ${ }^{9}$

$\left(B^{\prime}\right)$ Demgegenüber wird in der Standardökonomik der Wert in Gestalt der Nutzenschätzung möglicher Handlungsoptionen direkt als handlungsleitende Kategorie behandelt. Er ist der Antrieb für die Interaktion mit anderen Akteuren und schließt bei entsprechender Bewertung - die Nicht-Interaktion als Option ein. Im Gegensatz zum Marxschen Konzept gibt es daher keinen Tauschzwang. ${ }^{10}$ Damit verbunden ist eine Beschränkung auf die Betrachtung der individuellen Handlungslogik und de-

\footnotetext{
6 In dieser verdinglichten Form der gesellschaftlichen Anerkennung kann aus der Sicht des Marxsch en Konzepts die Hauptursache für den „Wachstumszwang“ gesehen werden: er ist ein Ergebnis des gesellschaftlichen Anerkennungswettbewerbs.

7 Es ist nicht klar, welche Bedeutung Hanappi (2019) der Arbeitstheorie des Werts bei Marx beimisst. Einerseits hält er die mit der simultanen Produktionstheorie mögliche präzise Arbeitszeitrechnung für sinnvoll (Hanappi 2019, S. 7), andererseits gibt er aber keine Antwort auf die Frage, welche analytische Bedeutung diese Arbeitszeitrechnung für die Behandlung der kapitalistischen Produktionsweise hat (Hanappi 2019, S. 10f.).

8 Diese von Marx implizit im „Kapital“ suggerierte Geschlossenheit der ökonomischen Analyse hat ihr standardökonomisches Pendant in der mit Märkten per se verbundenen „Allokationseffizienz“. Auf den ersten Blick scheinen daher in beiden Fällen politische Interventionen von untergeordneter Bedeutung für die ökonomische Reproduktion zu sein. Hanappi (2019) sieht dieses Problem hauptsächlich bei der Standardökonomik (Hanappi 2019, S. 4f.) nicht aber bei Marx.

9 Diese enge Verknüpfung der ökonomischen Reproduktion mit politischer Regulierung steht der beliebten Unterstellung entgegen, dass der Staat für Marx lediglich als Ausschuss für die Gemeinschaftsgeschäfte der Bourgeoisie zu behandeln sei (vergl. etwa Petersen und Faber 2013, S. 146ff.).

10 Die damit verbundenen theoriesystematischen Probleme sind von Georgescu-Roegen (1966) genauer diskutiert worden.
} 
ren Immunisierung gegen allfällige gesellschaftliche Einflüsse (homo oeconomicusKonzept). Daraus folgt, dass gesellschaftliches Handeln nur aus der einfachen Aggregierung (Summe) der individuellen Handlungen bestehen kann. Marx sieht diese Hypostasierung des Individuums selbst als ein ideologisches Produkt der modernen Warengesellschaft an (vergl. Marx 1983, S. 19f.) und damit als ein Beispiel für die Transformation eines speziellen historischen Sachverhalts in einen überhistorisch gültigen Sachverhalt.

Wie diese Interaktion mit anderen Akteuren jenseits bilateraler Beziehungen zustande kommt, kann auf Basis dieser (auf individuellen Zuschreibungen beruhenden) Wertlehre nicht erklärt werden; die dafür erforderlichen intersubjektiven (gesellschaftlich „objektiven“) Bezugsgrößen (wie etwa Preise oder Budgets) werden als gegeben vorausgesetzt. Die Analogisierung von „objektiven“ und subjektiven Bezugsgrößen gelingt daher nur auf Basis einer künstlichen Arithmetisierung der letzteren (etwa in Gestalt der Axiome für die Präferenzordnung). Insoweit wird den beobachtbaren intersubjektiven historisch-spezifischen ökonomischen Größen ein überhistorisches Handlungskonzept unterlegt und die ersteren dadurch legitimiert.

Diese Art von Werttheorie ist das Gegenprogramm zum Marxschen Wertkonzept: Es gibt keine Grundlage dafür, von einer Verdinglichung gesellschaftlicher Verhältnisse und deren Verselbständigung gegenüber den Akteuren auszugehen, da alle beobachtbaren ökonomischen Formen aus dem Zweck der Zielmaximierung der Akteure erklärt werden sollen und historisch spezifische Sachverhalte, die die gesellschaftliche Interaktion erst ermöglichen (wie etwa Geld und Budgets) in diese überhistorische Betrachtung eingeschmuggelt werden. Aus der Marxschen Perspektive muss die Standardökonomik daher als eine mathematisch (modelltheoretisch) verklausulierte Rechtfertigungslehre (Ideologie, die dem Bereich der ,Vulgärökonomie“ zuzurechnen ist) charakterisiert werden (vergl. Hanappi 2019, S. 22).

\subsection{Klassentheorie der Produktion und endogener technischer Wandel}

(C) In Gestalt der kapitalistischen Unternehmen behandelt Marx den institutionellen Kontext für das Akteurshandeln: hier entwickelt er, wie soziale Organisationsbildung und Implementierung von Technik spezifische Handlungserfordernisse für die beteiligten Akteure hervorbringen. ${ }^{11}$ Dies erfolgt in Gestalt einer Fortschreibung der in (A) skizzierten dualen Betrachtungsperspektive: durchgängig wird eine Unterscheidung und Relationierung der überhistorisch/universellen Elemente der Produktion und deren spezifisch historische Ausprägung in der kapitalistischen Fabrik vorgenommen.

Im Vordergrund steht dabei deren spezifisch historischer Charakter als kommodifizierte Ausbeutung: das kapitalistische Unternehmen kann Elemente kaufen bzw. nutzen, die mehr ökonomisches Anerkennungspotential (alias Werte) hervorbringen können als sie selber erfordern (Möglichkeit der Ausbeutung). ${ }^{12}$ Prominent bei Marx

\footnotetext{
${ }_{11}$ Wohl nicht zuletzt entsprechend der noch unentwickelten Dynamik im Konsumbereich behandelt Marx den institutionellen Kontext der individuellen oder familiären Reproduktion nur am Rande.

12 Dies ist in gewisser Weise die vorweg genommene Marxsche Antwort auf die Frage von Coase (1952), warum es überhaupt Unternehmen gibt.
} 
ist in diesem Zusammenhang die „Ware Arbeitskraft“, die - wenn's gut läuft - mehr Werte (bzw. realisierbare Preise) produzieren kann als sie selber kostet. Zumindest für diese Arbeitskraft gibt es die soziale Konnotation, dass sie nur dann existiert, wenn ihr selbst die Verwendung von Produktionsmitteln, also eigene Produktion verwehrt ist. Damit gibt es zwei Klassen von Warenbesitzern die im Unternehmen unter der Regie des (hier als Produktionsmitteleigentümer verstandenen) Kapitalisten aufeinandertreffen.

Diese Ausbeutung generell und speziell die sozial konnotierte Ausbeutung der Arbeitskraft prägen Produktionstechnik und -organisation im kapitalistischen Unternehmen: das planvolle Zusammenwirken der Elemente (Kooperation), deren Spezialisierung (Teilung der Arbeit) und die Installierung von automatisierten Abläufen (Maschinerie) dienen der Steigerung der Ausbeutung. Dies definiert in der Sicht von Marx die „Rationalität“ des Unternehmenshandelns. Die Veränderung von Produktionstechnik und -organisation ergibt sich für Marx aus der Steigerung dieser „Rationalität“: zum einen erzielen die Entrepreneur-Kapitalisten durch Steigerung der Produktivität und/oder der Qualität temporäre Extraprofite, zum andern sind sie bestrebt, die Veränderung von Produktionstechnik und -organisation als Instrument zur sozialen Subordination und insbesondere bei Lohnsteigerungen aufgrund von Arbeitskräfteknappheit deren Disponibilität aufrechtzuerhalten. Der auf diese Weise endogen erklärte technische Wandel dient der Sicherstellung bzw. Verbesserung der primären Einkommensverteilung zugunsten der Entrepreneur-Kapitalisten.

Marx geht davon aus, dass entweder aufgrund technischer Eigenheiten oder aufgrund des nur lokalen Wissens von Entrepreneur-Kapitalisten die Produktionstechnik und -organisation limitationalen Charakter aufweist (vergl. Marx 1976, S. 217). Der Sach- und Zeitbedarf entsprechender Veränderungen kann daher - modern gesprochen - in Form einer Angebotstrajektorie zusammengefasst werden. Für die Formulierung einer „Angebotsfunktion“ gibt es daher keine Grundlage. Die soziale Bestimmung des Konsums (sei es der Arbeitskräfte, sei es der Kapitalisten) wird nur am Rande behandelt. Marx folgt hier der klassischen Unterscheidung in den Konsum notwendiger Lebensmittel und dem Luxuskonsum. Abgesehen von dem Verweis auf ein von den Zeitumständen abhängiges ,historisch-moralisches“ Element in der Lohnzumessung (und den zyklischen Fluktuationen des Arbeitslohns) bleibt die Nachfragetrajektorie der Lohnbezieher unbestimmt.

$\left(C^{\prime}\right)$ Dieser Sichtweise steht in der Standardökonomik die Naturalisierung und historische Neutralisierung der Produktions- bzw. Konsumtätigkeit gegenüber. Deren Veränderung kann dann nur durch exogene Faktoren erklärt werden. Die konkreten historischen institutionellen Binnenstrukturen und sozialen Einbettungen der ökonomischen Akteure (Unternehmen und private Haushalte) werden zugunsten abstrakter funktionaler Stilisierungen der Handlungsoptionen (Produktionsfunktion vs. Nutzenfunktion) weitgehend ignoriert. Obwohl deren Handeln auf einen Marktkontext bezogen sein soll, werden die damit verbundenen prekären Anerkennungsprozeduren und die dabei zum Tragen kommenden sozialen Asymmetrien ausgeblendet. Die Produktionstätigkeit ebenso wie die Konsumtätigkeit erscheint insoweit weitgehend technisch bzw. allgemein anthropologisch bestimmt zu sein.

Sowohl bei der Produktion als auch beim Konsum wird davon ausgegangen, dass durch das Handeln der Akteure die jeweiligen Elemente so eingesetzt wer- 
den, dass deren (als beobachtbar unterstellter) Grenzbeitrag zum Handlungsergebnis (produzierte Gütermengen bzw. erzielte Nutzeneffekte) dem Preis für diese Elemente entspricht. Dies korrespondiert einem internen optimalen Gleichgewichtszustand bei den ökonomischen Akteuren. Die für den Marktkontext üblichen Veränderungen und Innovationen im Handeln der Akteure können daher nur durch exogene Faktoren bedingt sein (im Falle der Unternehmen: neue technische Erkenntnisse bzw. Faktorpreisänderungen). Weder die ontologische Triftigkeit dieser Annahmen noch die informationellen Voraussetzungen für ihre handlungspraktische Wirksamkeit werden dabei plausibel gemacht sodass diese Charakterisierung der institutionellen Binnenstruktur der ökonomischen Akteure nur als Fiktion qualifiziert werden kann. Gleichwohl stellt sie eine mathematisch rationalisierte Variante der „Faktortheorie“ dar die Marx als höchste Form der verdrehten ökonomischen Erscheinungen charakterisiert hat (vergl. Marx 1992, S. 834 ff.).

\subsection{Umrisse einer ökonomischen Entwicklungstheorie}

(D) In dem von Marx hinterlassenen ökonomietheoretischen Werk können nur Umrisse bzw. Fragmente einer umfassenden ökonomischen Entwicklungstheorie identifiziert werden. In diesen Fragmenten werden nur einzelne Aspekte einer derartigen Entwicklungstheorie behandelt, weil sie auf den verschiedenen Darstellungsstufen unterschiedlichen Erklärungszwecken dienen, als solche unfertig und nicht miteinander systematisch verknüpft sind. ${ }^{13}$

Den Ausgangspunkt bildet die auf der Darstellung der Ausbeutung in der Produktion aufbauende Darstellung der „Akkumulation des Kapitals“ im 7. Abschnitt des ersten Bandes des „Kapital“ (vergl. Marx 1987, S. 561 ff.). Hier wird verdeutlicht, wie sich die soziale Subordination der Lohnarbeiter im Produktionsprozess und die damit angestrebte Verschiebung der primären Einkommensverteilung zugunsten der Entrepreneur-Kapitalisten gebunden ist an die durch eine Umstrukturierung der Produktionstechnik erfolgende Herstellung entsprechender Konstellationen auf dem Markt für Arbeitskräfte. Die dafür erforderliche relative Überflüssigmachung eines Teils der letzteren stellt sich in einer zyklischen Veränderung der Einkommensproportionen her.

In den posthum veröffentlichten Manuskripten zum zweiten Band des Kapitals (vergl. Marx 2008) werden zum einen die sachlichen und zeitlichen Strukturerfordernisse des Kapitals in seinem „Kreislauf“ dargestellt. Dabei wird deutlich, wie die Dauer und Zusammensetzung des Umschlags des Kapitals sowie die Verknüpfung von zirkulierendem mit dem fixen Kapital Anforderungen für die Aufteilung des Kapitals auf die verschiedenen Formen des Warenkapitals, des produktiven Kapitals und des Geldkapitals stellen, wenn der „Kreislauf“ des Einzelkapitals flüssig vonstatten gehen soll. Zum andern wird in diesen Manuskripten unter stilisierten Bedingungen gezeigt, wie für ein gegebenes in Warenform vorliegendes Gesamtkapital ein gesellschaftlicher Umsatz so möglich ist, dass die stofflichen und wertmäBigen Bedingungen für den Fortgang des „Kreislaufs“ aller Kapitale hergestellt wer-

13 In der Literatur gibt es m. W. bisher keine Versuche, alle diese Fragmente systematisch zu rekonstruieren und zu synthetisieren. 
den. Dieser Betrachtung werden Wertaggregate stofflich-funktional unterschiedener „Abteilungen“ der Industrie (im Wesentlichen: Produktionsmittel herstellende und Konsummittel herstellende Industrien) zugrundegelegt und die Bilanzierung bzw. Ersetzung der Elemente dieser Wertaggregate soll durch Geld vermittelt werden (Reproduktionsschemata).

Schließlich finden sich in den Manuskripten zum dritten Band des „Kapital“ Skizzen für weitere Spezifikationen der Reproduktionstheorie. Dies sind zum einen Überlegungen zur langfristigen Entwicklung der Kapitalakkumulation in Gestalt des sich hinterrücks herstellenden ,tendenziellen Falls der Profitrate“ (vergl. Marx 1992, S. 285 ff.) - ohne dass allerdings die im ersten Band behandelten Zusammenhänge zwischen der Veränderung der Primärverteilung (Mehrwertrate) und der Veränderung der Kapitalzusammensetzung (organische Zusammensetzung des Kapitals) systematisch aufgenommen werden. ${ }^{14}$ Zum andern wird die eher kurz- und mittelfristige Analyse der zyklischen Kapitalakkumulation angereichert durch die Einbeziehung des Kreditsystems, für das der „Kreislauf“ der Kapitale zwar die Grundlage darstellt, das sich aber - bedingt durch die Ausbildung einer eigenständigen Sorte von Geldkapitalisten und von Banken - zu einem künstlichen Verstärker für die industrielle Zyklik entwickelt (vergl. Marx, 1992, S. 469 ff.).

$\left(\mathrm{D}^{\prime}\right)$ Der Fokus des Theoriekonzepts der neoklassischen Standardökonomik liegt auf der „Allokation knapper Ressourcen“. Die für eine Entwicklungstheorie zentrale Veränderung des Ressourcenbestandes ebenso wie der Bedürfnisse über die Zeit ist damit aus systematischen Gründen ausgeblendet (oder unterbelichtet). Allenfalls Pfade für deren gleichgewichtiges Wachstum werden thematisiert (Solow, Ramsey). Die sog. ,neue Wachstumstheorie“ zeigt, dass die Integration entrepreneurkapitalistischer Innovationen in das neoklassische Theoriegebäude nur um den Preis einer konzeptionellen Inkonsistenz erfolgen kann. Zugespitzt formuliert: die übliche produktionstheoretische Stilisierung (etwa in Gestalt einer Cobb-Douglas Produktionsfunktion) in einem Wettbewerbssektor wird um einen eigenständigen risikofreien F\&E Sektor und einen intermediären Sektor, der dessen Ergebnisse quasimonopolistisch verwerten kann, ergänzt.

Insoweit lässt sich folgern, dass die von Marx behandelten Aspekte einer ökonomischen Entwicklungstheorie von der Standardökonomik nicht behandelt werden und nach wie vor eine Herausforderung für die ökonomischen Forschung darstellen.

\subsection{Umrisse einer prozeduralen Markt- und Wettbewerbsanalyse}

(E) Märkte sind in der Marxschen Perspektive der Bereich, in dem - entsprechend des werttheoretischen Ansatzes - die Anerkennungsansprüche der privaten Akteure aufeinandertreffen und insoweit auch Unsicherheit, soziale Rivalität und die Artikulation von Machtansprüchen ihren Ort haben. Entsprechend seiner Erklärungsmethode legt Marx zum Marktgeschehen eine mehrstufige, immer konkreter (spezifizierter) werdende, Analyse vor (vergl. Beckenbach 2015). Zentrale Aufgabe dieser Analyse

14 Dies ist nur im Rahmen einer dynamischen Analyse möglich. In einem derartigen (für Marx noch nicht zugänglichen) Analyserahmen lässt sich dieses „Gesetz“ - entgegen der vollmundig vorgetragenen Kritik in der Literatur - konsistent reformulieren. Davon unbenommen ist die Frage der empirischen Triftigkeit. 
ist die Erklärung der die beobachtbaren Marktoperationen (v.a. Preise aber auch Mengen und Marktanteile) regulierenden Sachverhalte.

Bereits auf der abstraktesten Ebene der ,einfachen Warenzirkulation“ wird deutlich, dass das Regulativ für die Marktpreise ein sich aus heterogenen Elementen zusammensetzendes Aggregat ist: die Preise sollen über einen längeren Zeitraum entsprechend der o.g. tauschtheoretischen Hypothese - durch die ,,gesellschaftlich notwendige Arbeitszeit" reguliert werden (einfache Wertpreise). Eine Gesamtheit der Produzenten einer bestimmten Ware wird dabei in Abhängigkeit von ihrem jeweiligen Produktionsumfang und ihrer jeweiligen Produktivität als Einflussgröße herangezogen und damit - entsprechend des sozialen Kontextes - als ein selbst veränderliches $\mathrm{Maß}$ unterstellt. ${ }^{15}$

Nach der Analyse des kapitalistischen Produktionsprozesses und der expliziten Einbeziehung der Vielheit der Kapitale in der Konkurrenz wird klar, dass die für die Preisregulation erforderliche Betrachtung nicht nur die beständige Unterscheidung unterschiedlich großer und unterschiedlich produktiver Kapitale (für die Produktion einer gegebenen Warenart) erfordert, sondern auch eine fortwährende Veränderung dieser „Population“ in Rechnung zu stellen ist, da stets neue produktivere und/ oder qualitätsvollere Produktionsmethoden eingeführt werden, sich auf dem Markt ausbreiten und weniger produktive bzw. weniger qualitätsvolle Produktionsmethoden verdrängen. Auskunft darüber zu geben, welches Gewicht welche Produzentengruppe bei der Regulierung der Preise hat, ist dann ebenso eine Aufgabe der Analyse des Marktwerts wie die Berücksichtigung des Einflusses des Verhältnisses von Angebots- und Nachfragegrößen, die sich aus den Reproduktionserfordernissen ergeben. ${ }^{16}$ Das Austarieren dieser Einflussfaktoren zu einer regulierenden Marktwertgröße schließt zwar entsprechend der Heterogenität der Produzenten Verwertungsdifferentiale (,Marktrenten“) ein, diese sind aber im Prinzip für einen gegebenen Produzenten nur temporär erzielbar.

Auf Basis dieser Ausgleichsprozesse innerhalb einer Industrie lassen sich für diese entsprechend Industrieprofitraten ermitteln, die sich je nach den Bedingungen der Produktion unterscheiden werden. Dies ist die Grundlage für die Relationierung der Industrien selber indem dauerhafte Unterschiede in den Industrieprofitraten einen Anlass für die Ab- bzw. Zuwanderung von Kapital schaffen. Diese Ausgleichsprozesse begründen eine Entwicklung in Richtung auf die Marktproduktionspreise als Regulativ für die Preise. Technologische Abgleichung, Angebots- und Nachfragebedingungen sowie $\mathrm{Zu}$ - bzw. Abwanderungsprozesse des Kapitals werden damit von Marx als die entscheidenden Faktoren für die Regulation der Preise identifiziert.

Diese mehrstufige Form der Erklärung des Regulativs für die Marktpreise beruht auf der Überlegung, dass jeweils die Gesamtheit der Produzenten einer bestimmten

\footnotetext{
15 Insofern folgt Marx der Leitidee, dass es ein ,,inneres“ Preismaß gibt, dieses aber - im Unterschied zu Maßen in naturwissenschaftlichen Kontexten - variabel ist. Nach einem invarianten Preismaß zu suchen ist Ricardo und seinen modernen Nachfolgern vorbehalten.

16 Marx thematisiert hier v. a. kurzfristig nicht abbaubare Differenzen zwischen den sich aus der Reproduktion ergebenen Angebots- und Nachfragegrößen; dabei werden jeweils diskrete Einzelkonstellationen und keine Angebots- und Nachfragefunktionen unterstellt.
} 
Warenart dieses Regulativ beeinflusst. ${ }^{17}$ Gibt es nun aber nicht verallgemeinerbare Produktionsbedingungen, die nur für einen Teil dieser Produzenten zugänglich sind, dann definieren Teilgruppen dieser Produzenten dauerhaft das Regulativ für die Marktpreise. Die Grenzen für die Verallgemeinerung können entweder in natürlichen Produktionsbedingungen begründet sein (etwa Grund und Boden, sonstige natürlichen Ressourcen) (Grenzpreis) oder in einer Dominanz eines oder mehrerer Produzenten auf dem betreffenden Markt (Monopolpreis). In beiden Fällen werden die Nachfrage und deren Preiselastizität zu einem zentralen Einflussfaktor für den Preis und die „Marktrenten“ zu einem Dauerphänomen.

$\left(\mathrm{E}^{\prime}\right)$ Eigentlich sind Märkte der zentrale Koordinationsmechanismus der ökonomischen Aktivitäten in der Standardökonomik. Sie werden aber so konstruiert, dass die ihre individuellen Ziele verfolgenden Akteure in einer Weise koordiniert werden, dass für alle Akteure ein Maximum an Zielerreichung gegeben ist (Gleichgewichtspostulat). Da die Preise, die dieses sicherstellen, entsprechend des Konzepts des „,vollständigen Wettbewerbs“ nicht selber durch die Marktakteure hervorgebracht werden können, ist der Markt hier de facto ein Planungsinstrument zur aggregierten individuellen Zielmaximierung. Wechselseitige Abhängigkeiten der Akteure sind in diesem Rahmen nur eingeschränkt abbildbar (für Kleingruppen etwa in spieltheoretischen Ansätzen). Die mit diesen Abhängigkeiten grundsätzlich zusammenhängenden Unsicherheiten, sozialen Rivalitäten und Machtausübungen haben hier keinen Platz. Das Wesen des Marktes als einer spezifisch historischen Interaktionsform von Warenproduzenten, die dort ihre gesellschaftlichen Geltungsansprüche explorieren und exploitieren, wird damit ausgeblendet.

Dieses Planungskonzept des Marktes wird in unterschiedlicher Weise spezifiziert. In der partialanalytischen Betrachtung der Mikroökonomik entweder direkt als Konstruktion und Relationierung von Angebots- und Nachfragefunktionen oder als Ableitung dieser Funktionen aus den Zielfunktionen von Unternehmen und privaten Haushalten. In der totalanalytischen Betrachtung der Mikroökonomik (Berücksichtigung der Interdependenz aller Märkte) erfolgt dies in Gestalt von fiktiven Regulatoren (Auktionator) oder in Gestalt von marktfremden Beschränkungen (spezifische Ausstattungserfordernisse, Dominanz des Substitutionseffektes über den Einkommenseffekt, Bruttosubstituierbarkeit usw.). Während in der Mikroökonomik die Heterogenität der Akteure noch rudimentär in Gestalt von Ausstattungsunterschieden bzw. Unterschieden in den jeweiligen Zielfunktionen berücksichtigt werden können, ist in der Makroökonomik diese Heterogenität in der Unterstellung „,repräsentativer Akteure" aufgehoben. Diese werden in der älteren Makroökonomik (z.B. IS-LM Modell) zur Grundlage von subjektlosen funktionalen Aggregatbeziehungen, die für die verschiedenen Märkte so konstruiert werden, dass nur gesamtwirtschaftliche Gleichgewichte existieren. In der neueren Makroökonomik hingegen darf sich dieser repräsentative Akteur - ausgestattet mit ,rationalen Erwartungen“ - in einer exogen generierten turbulenten Umgebung als Gleichgewichtsgenerator bewähren (DSGEModelle).

17 Marx operiert überwiegend mit der Idee der Durchschnittsbildung, was bei schiefen bzw. mehrgipfligen Verteilungen problematisch ist. 
Dem Marxschen Versuch, auf unterschiedlichen Abstraktionsebenen in den Marktprozessen die Regelzentren für die Marktpreise zu identifizieren und dabei die Spezifika der kapitalistischen Warenproduktion zu berücksichtigen (Heterogenität der Akteure und Industrien, unterschiedliche zeitliche Skalierung der Regelprozesse, hinterrücks sich herstellende Regelzentren fern vom Gleichgewicht) steht in der Standardökonomik die modelltheoretisch untermauerte Abschaffung des Marktes als sozialer Form gegenüber. Stattdessen werden Maximierungsautomaten von einem sichtbaren Regulator (statt einer unsichtbaren Hand) auf ein Koordinationsgleichgewicht verpflichtet. Der von Marx diagnostizierte Fetischismus der ökonomischen Formen (hier: die hinterrücks ergebende ordnende Rolle der Warenpreise) wird dabei auf eigentümliche Weise modelltheoretisch rationalisiert (vergl. Leontief 1966, S. $72 \mathrm{ff}$.).

\section{Anforderungen für eine Modellierung des Marxschen Konzepts}

Aus dieser Kurzrevue der wichtigsten Botschaften der Marxschen „Kritik der Politischen Ökonomie“ lassen sich als wichtigste Anforderungen für eine Modellierung des Marxschen Konzepts ableiten:

i. Die Berücksichtigung der Differenzierung ökonomischer Tätigkeiten und daher das Erfordernis eines gesellschaftlichen Syntheseprozesses (bzw. Aggregation). Damit verbunden ist die zentrale Rolle von Preisen als Explorationsmedien und des Geldes als sozialer Moderationsinstitution.

ii. Die Einbeziehung des Antagonismus auf der Ebene der primären Einkommensverteilung (in terms of „Lohn“ und „Profit“)

iii. Die Abbildung der Indienstnahme des technischen Wandels für die Veränderung dieser Einkommensverteilung und die dadurch erfolgende Strukturierung des Marktes für Beschäftigungsgelegenheiten (,Arbeitsmarkt“)

iv. Die Erfassung der Eigentümlichkeiten des Reproduktionsprozesses im Spannungsfeld von Marktprozessen und Reproduktionsanforderungen.

Die für die modelltheoretische Präzisierung des Marxschen Konzepts oft (und auch von Hanappi 2019) verwendeten Modellvarianten sind das - auf Ricardo zurückgehende - Kornmodell, das von Sraffa entwickelte lineare Produktionsmodell und das der mathematischen Populationsökologie (v. a. Lotka- Volterra) entstammende zyklische Wachstumsmodell. Es ist erstaunlich, dass keines dieser Modelle alle o.g. essenziellen Merkmale des Marxschen Konzepts abbilden kann. Das Merkmal (ii) wird im Kornmodell und auch im linearen Produktionsmodell aufgenommen, die Merkmale (ii) und (iii) werden im zyklischen Wachstumsmodell behandelt. Die Merkmale (i) und (iv) werden in keiner dieser Modellvarianten erfasst.

Der Hintergrund für diesen bedauerlichen Befund kann einerseits gesehen werden in einer Prägung der ökonomischen Modellierung durch das statische Gleichgewichtskonzepte auch dort, wo nicht die soziale Harmonie die Leitlinie für die gewählten Fragestellungen (gewesen) ist (wie dies in der Neoklassik der Fall ist). Offensichtlich sollte das Sichtbarmachen sozialer Antagonismen dadurch an (wissenschaftlicher) Überzeugungskraft gewinnen, dass sie in einen gleichgewichtstheo- 
Abb. 1 Inverse Beziehung zwischen Lohnsatz (w) und Profitrate (r) im Kornmodell (a) und im linearen Produktionsmodell (b)
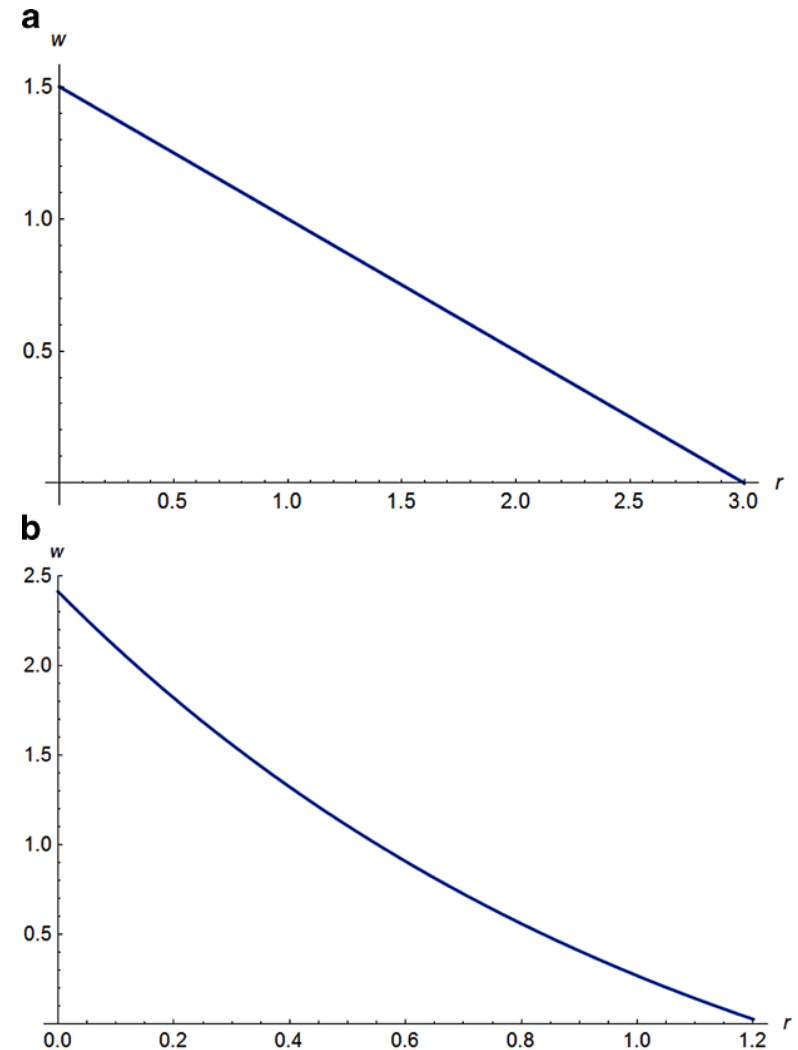

retischen Rahmen präsentiert wurden. Dies zeigt sich sowohl im Kornmodell als auch im linearen Produktionsmodell indem auf Basis einer gegebenen - als reproduktionsfähig angenommenen - Technologie der Antagonismus der primären Einkommensverteilung (gemessen in Gewichtseinheiten Korn bzw. einer als Maßstab unterstellten Ware) als inverses Verhältnis von Profitrate (r) und Lohnsatz (w) dargestellt wird (vergl. Abb. 1). ${ }^{18}$

Gemessen an den Modellierungserfordernissen, die sich aus dem Marxschen Konzept ableiten lassen, weisen diese Modellvarianten aber gravierende Mängel auf (die darauf verweisen, dass diese Modellarchitekturen in einem anderen Kontext entstanden sind):

- Die Fokussierung des sozialen Antagonismus erfolgt in Gestalt einer extern festgelegten Verteilungsvariablen die unabhängig von der Technologie ist (und daher bei gegebener Technologie auch variieren kann). Damit ist sowohl die Variation der

\footnotetext{
18 Anders als bei Marx wird dabei unterstellt, dass die Lohnkosten nicht Teil des vorgeschossenen Kapitals sind. Im Kornmodell ist dann dieser Zusammenhang zwischen Profitrate und Lohnsatz linear, weil Maßstab und Messobjekt dieselbe Qualität aufweisen; im linearen Produktionsmodell ist dieser Zusammenhang dann nicht - linear, wenn die als Maßstab fungierende Ware eine andere Struktur aufweist als das Messobjekt.
} 
Technologie über die Zeit ausgeblendet als auch der Zusammenhang dieser Variation mit der Veränderung des sozialen Antagonismus wie Marx das thematisiert. ${ }^{19}$

- Es gibt keine Preise (und damit auch keine Marktprozesse und Geld): die ,relativen Preise" sind von außen festgelegte Proportionierungsfaktoren für die verschiedenen Industrien, die die geforderten Gleichverteilungsansprüche der verschiedenen Industrien sicherstellen sollen. ${ }^{20}$

Essenzielle Merkmale der kapitalistischen Produktion wie Marx sie vor Augen hatte, werden damit nicht erfasst.

Andererseits kann als Hintergrund für den obigen Modellbefund der Umstand angesehen werden, dass es in Bezug auf die eigentlich erforderliche formal-mathematische dynamische Analyse keinen theoriegeschichtlichen Vorlauf in der Ökonomik gegeben hat. Insofern ist es nicht verwunderlich, dass hier Beleihungen in der Populationsökologie vorgenommen wurden in der diese Analyseform früh entstanden ist (vergl. Kingsland 1985). Nicht zuletzt aufgrund der Sicherstellung der mathematischen Handhabbarkeit wird in diesen dynamischen Modellierungen von in sich homogenen Populationsarten (die jeweils durch ihre Anzahl gemessen werden können) ausgegangen. Die Sicherstellung eindeutiger Resultate bei der Verwendung dieses Ansatzes in der Ökonomik erfordert daher die Ausschließung von Messambivalenzen wie sie etwa in der linearen Produktionstheorie bei Berücksichtigung mehrerer technologisch heterogener Industrien auftreten. Der „Preis“ für den Wechsel zur dynamische Analyse (und damit auch für die zumindest implizite Berücksichtigung des technischen Wandels und seiner Bedeutung für die primäre Einkommensverteilung) ist insofern - analog zum Kornmodell - der Rückgang auf die Betrachtung einer (,repräsentativen“) Industrie.

Auch wenn dieser Wechsel in einen dynamischen Modellierungsrahmen einen Fortschritt darstellt (da würde ich Hanappi 2019 zustimmen), bleiben wichtige Desiderata für eine vom Marxschen Konzept inspirierte Modellierung unerfüllt:

- Zunächst ist - nicht zuletzt angesichts der weiteren Diskussion in der mathematischen Populationsökologie - darauf zu verweisen, dass hier ein sehr spezieller Algorithmus für ein „konservatives“ dynamischen System verwendet wird, bei dem durch die Vorgabe eines Startwerts eine unveränderliche zyklische Trajektorie definiert ist. Demgegenüber ist mit dem allgemeineren Ansatz für die Modellierung

\footnotetext{
19 In der Diskussion des Kornmodells (vergl. Hanappi 2019, S. 13 f.) wird dieser Mangel besonders eklatant, indem die für die Sicherstellung des (fälschlicherweise Marx unterschobenen) Minimallohns erforderlichen Prozesse der Verfügbarmachung von Arbeitskraftreserven (insbesondere wenn das Modell als Wachstumsmodell reformuliert wird) gar nicht auftauchen.

20 Dass damit keine ,realen“ Preisbildungsprozesse abgebildet werden, kann auch aus dem Umstand abgeleitet werden, dass diese ,relativen Preise“ je nach der vom Modellierer verfolgten Lösungsstrategie für das Modell normiert werden können. Im Kornmodell werden eigentlich nur Gebrauchswertmengen zwischen dem landwirtschaftlichen Produzenten und den beschäftigten landwirtschaftlichen Arbeitern aufgeteilt. Warum der erstere unter diesen Bedingungen nur das Korn (als Saatgut) und nicht auch die anderen ökologischen Elemente wie Nährstoffe, Wasser usw. kalkulieren sollte, bleibt schleierhaft (vergl. Marx 1979, S. 1271f.). Eine derartige Selektivität in der Kalkulation wird erst auf Basis einer entsprechenden Selektivität eines Preissystems nachvollziehbar. Dessen Existenz setzt aber die Existenz entsprechender Märkte voraus.
} 

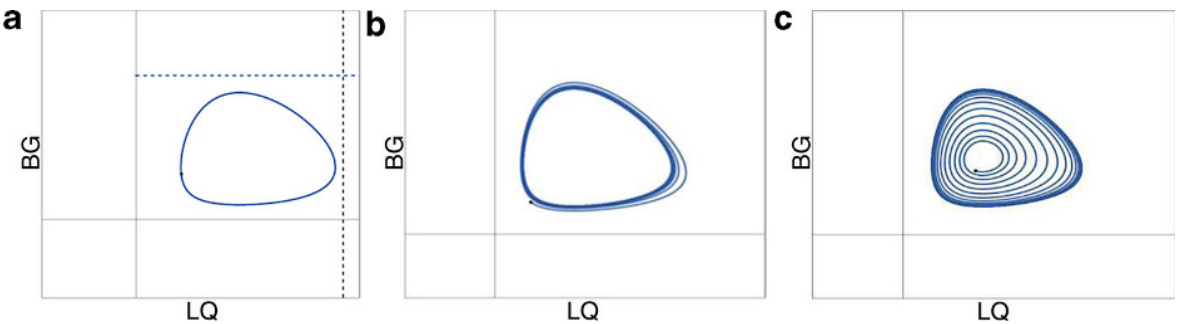

Abb. 2 Phasenraumdarstellung der zyklischen Entwicklung von Lohnquote (LQ) und Beschäftigungsgrad (BG) in einem konservativen Lotka/Volterra-Modell (a) und in einem dissipativen Systemmodell mit unterschiedlichen Startwerten (b und c)

„dissipativer“ Systeme (vergl. May 1974 und Beckenbach 2001, S. 119ff.) die Möglichkeit gegeben, die zeitabhängige Veränderung von Trajektorien und deren Anziehung und Abstoßung durch Regulatoren im Phasenraum (Attraktor bzw. Repellor) zu berücksichtigen (vergl. Abb. 2). Vor dem Hintergrund der Marxschen Idee, dass der Wert sich ,hinterrücks“ als Regulator durchsetzt, könnte dies eine zeitgemäße Alternative zur Fundierung des Werts durch Arbeit(smengen) sein. Darüber hinaus lassen sich in diesem Modellierungsrahmen Prozesse, die multiplen Regulatorentypen unterworfen sind (wie dies in der Marxschen Wettbewerbsanalyse gegeben zu sein scheint), abbilden.

- In der von Goodwin (1973) vorgenommen Spezifikation des konservativen Populationsmodells wird - wie im Kornmodell - nur ein Sektor betrachtet und die zyklische Entwicklung zwischen der Lohnquote (LQ) und dem Beschäftigungsgrad (BG) modelliert. Dabei wird nicht explizit erfasst, wie sich durch welche technischen Veränderungen der Beschäftigungsgrad im Sinne des Marxschen Konzepts verringern lässt; mit der Vorgabe des zyklischen Algorithmus wird unterstellt, dass dies immer erfolgreich von statten gehen kann (vergl. Hanappi 2019, S. 15). Darüber hinaus spielen in einem derartigen vereinfachten Modellkontext Preise, Geld sowie Marktprozesse - wie auch in den anderen hier behandelten Modellvarianten - keine Rolle.

Als Ergebnis dieser Modelldiskussion lässt sich festhalten: Es gibt entweder die Berücksichtigung sozialer Antagonismen im statischen Gleichgewichtsrahmen mit einer Welt aus heterogenen Industrien oder die Berücksichtigung sozialer Antagonismen in einem dynamischen Kontext mit einer Welt aus einer homogenen Industrie. In keinem Fall spielen Preise, Geld und Marktprozesse bzw. die Reproduktionserfordernisse in einer Welt mit heterogenen Industrien eine Rolle wie Marx dies in den Fragmenten zu seiner Entwicklungs- und Wettbewerbstheorie angemahnt hatte. Es erscheint daher völlig unangemessen, das Marxsche Konzept vorwiegend aus der Sicht dieser speziellen Modellierungsvarianten zu beurteilen. ${ }^{21}$ Die große Diskre-

\footnotetext{
21 Ein Beispiel dafür ist das „Transformationsproblem“, das vorwiegend aus der Sicht der linearen Produktionstheorie formuliert wird (vergl. den Überblick bei Beckenbach 2018, S. $44 \mathrm{ff}$.). Das wird auch von Hanappi (2019) konzediert (Hanappi 2019, S. 10), ohne dass allerdings der dafür herangezogene modelltheoretische Rahmen von ihm explizit problematisiert wird.
} 
panz zwischen den Merkmalen des Marxschen Konzepts und den Fokussierungen der gängigen Modellierungen sollte vielmehr Anlass für weitere Forschungsanstrengungen sein.

\section{Literatur}

Beckenbach, F. (2001). Beschränkte Rationalität und Systemkomplexität: Ein Beitrag zur ökologischen Ökonomik. Marburg: Metropolis.

Beckenbach, F. (2018). Warenproduktion mittels Waren? Eine kritische Würdigung des ökonomietheoretischen Ansatzes von Marx. In G. R. Lucas, R. Pfriem \& C. Thomasberger (Hrsg.), Auf der Suche nach dem Ökonomischen - Karl Marx zum 200. Geburtstag, S. 43-81). Marburg: Metropolis-Verlag.

Beckenbach, F., et al. (2016). Zur Pluralität der volkswirtschaftlichen Lehre in Deutschland - Eine empirische Untersuchung des Lehrangebots in den Grundlagenfächern und der Einstellung der Lehrenden. Marburg: Metropolis.

Beckenbach, F. (2017). Die (Re)Produktion der modernen Standardökonomik als Problem für eine transformative Umorientierung. In E.R. Pfriem et al (Hrsg.), Transformative Wirtschaftswissenschaft im Kontext nachhaltiger Entwicklung (S. 165-212). Marburg: Metropolis-Verlag.

Beckenbach, F. (2015). Artikel „Marktpreis“. Historisch-Kritisches Wörterbuch des Marxismus. W. F. Haug, F. Haug, P. Jehle and W. Küttler. Hamburg, Argument-Verlag. 8/II: 1784-1793.

Coase, R.H. (1952). The Nature of the Firm. Readings in Price Theory. R. D. Irwin. Homewood/Ill (S. 331-351).

Georgescu-Roegen, N. (1966). Analytical Economics-Issues and Problems. Cambridge: Havard University Press.

Goldstein, J. P. (2006). „Marxian Microfoundations: Contribution or Detour?“. Review of Radical Political Economics, 38, 569-594.

Goodwin, R. (1973). In E. K. Hunt \& J.G. Schwartz (Hrsg.), A Growth Cycle. A Critique of Economic Theory (S. 442-449). Harmondsworth: Penguin.

Hanappi, H. (2019). ,(Neo-)Marxistische Politische Ökonomie als Gegenprogramm zur Standardökonomik“. List Forum für Wirtschafts- und Finanzpolitik. https://doi.org/10.1007/s41025-019-00127-6

Kingsland, S.E. (1985). Modeling Nature: Episodes in the History of Population Ecology. Chicago: University of Chicago Press.

Leontief, W. (1966). The Significance of Marxian Economics for Present (S. 72-83). Economic Theory. Essays in Economics-Theories and Theorizing. W. Leontief. White Plains, International Arts and Sciences Press: Day.

Lippi, M. (1979). Value and Naturalism in Marx. London: New Left Books.

Marx, K. (1969). Randglossen zum Programm der deutschen Arbeiterpartei. In: Marx-Engels Werke. Bd. 19 (S. 15-32). Berlin: Dietz.

Marx, K. (1974). Brief an Kugelmann vom 11.7.1868. In: Marx-Engels Werke. Bd. 32 (S. 552-554). Berlin: Dietz.

Marx, K. (1983). Einleitung. In: Marx-Engels Werke. Bd. 42 (S. 19-45). Berlin: Dietz.

Marx, Karl (1976): Zur Kritik der Politischen Ökonomie (Manuskript 1861-1863), Teil 2. In: Marx, Karl; Engels, Friedrich. Gesamtausgabe (MEGA). MEGA II/3.2. Berlin, Dietz.

Marx, Karl (1979): Zur Kritik der Politischen Ökonomie (Manuskript 1861-1863), Teil 2. In: Marx, Karl; Engels, Friedrich. Gesamtausgabe (MEGA). MEGA II/3.4. Berlin, Dietz.

Marx, Karl (1987): Das Kapital. Kritik der Politischen Ökonomie. Erster Band, Hamburg 1872. In: Marx, Karl; Engels, Friedrich. Gesamtausgabe (MEGA). MEGA II/6. Berlin, Dietz.

Marx, Karl (1992): Ökonomische Manuskripte 1863-1867, Teil 2. In: Marx, Karl; Engels, Friedrich. Gesamtausgabe (MEGA). MEGA II/4.2 Berlin, Dietz.

Marx, Karl (2008): Manuskripte zum zweiten Buch des „Kapitals“ 1868-1881. In: Marx, Karl; Engels, Friedrich. Gesamtausgabe (MEGA). MEGA II/11. Berlin, Dietz.

May, R. M. (1974). Stability and Complexity in Model Ecosystems. Princeton: Princeton University Press.

Petersen, T., \& Faber, M. (2013). Karl Marx und die Philosophie der Wirtschaft - Bestandsaufnahme, Überprüfung, Neubewertung. Freiburg: Verlag Karl Alber. 